PROCEEDINGS OF THE

AMERICAN MATHEMATICAL SOCIETY

Volume 138, Number 3, March 2010, Pages 837-845

S 0002-9939(09)10121-1

Article electronically published on October 22, 2009

\title{
WHEN WEAK HOPF ALGEBRAS ARE FROBENIUS
}

\author{
MIODRAG CRISTIAN IOVANOV AND LARS KADISON
}

(Communicated by Martin Lorenz)

\begin{abstract}
We investigate when a weak Hopf algebra $H$ is Frobenius. We show this is not always true, but it is true if the semisimple base algebra $A$ has all its matrix blocks of the same dimension. However, if $A$ is a semisimple algebra not having this property, there is a weak Hopf algebra $H$ with base $A$ which is not Frobenius (and consequently, it is not Frobenius "over" $A$ either). Moreover, we give a categorical counterpart of the result that a Hopf algebra is a Frobenius algebra for a noncoassociative generalization of a weak Hopf algebra.
\end{abstract}

\section{INTRODUCTION}

Quasi-Hopf algebras are objects generalizing Hopf algebras, which were introduced in 1990 by Drinfeld. Quasi-Hopf algebras are associative algebras $H$ having a coalgebra structure which is only coassociative up to conjugation by a nonabelian 3-cocycle and which, together with an antipode $S: H \rightarrow H$, satisfy appropriate compatibility conditions. Weak Hopf algebras were introduced and investigated by several authors ( $\overline{\mathrm{BoSz}}, \overline{\mathrm{BNS}}, \mathbf{\mathrm { N }}, \overline{\mathrm{S}}$ ) ) and are of great interest in physics (e.g. BoSz, FFRS).

Finite dimensional Hopf algebras generalize group algebras of finite groups in many ways, and one of the interesting similar features they have is that they are Frobenius algebras. This property is also preserved in the infinite dimensional case, in the sense that an infinite dimensional Hopf algebra having a nondegenerate integral is co-Frobenius (as a coalgebra). It is then natural to investigate whether the property of being a Frobenius algebra is preserved for generalizations of Hopf algebras. This was shown to be true for finite dimensional quasi-Hopf algebras; even infinite dimensional co-quasi Hopf algebras are co-Frobenius as shown in [BC].

In addition, the paper $\mathrm{V}$ ] seems to show that finite dimensional weak Hopf algebras are Frobenius algebras. This was done as a consequence of the integral theory the author developed for weak Hopf algebras. However, G. Böhm noticed a gap in one of the key ingredients used for proving this fact in [V] p. 485]. But

Received by the editors November 20, 2008, and, in revised form, July 15, 2009.

2000 Mathematics Subject Classification. Primary 18D10; Secondary 16W30, 16S50, 16D90, $16 \mathrm{~L} 30$.

Key words and phrases. Weak Hopf algebra, quasi-Hopf algebra, Frobenius algebra, quasiFrobenius algebra, tensor category, Tannakian reconstruction.

The first author was partially supported by contract no. 24/28.09.07 with UEFISCU "Groups, quantum groups, corings and representation theory" of CNCIS, PN II (ID_1002).

(C)2009 American Mathematical Society Reverts to public domain 28 years from publication 
it remains true that weak Hopf algebras are quasi-Frobenius, which was proved in [V] and in BNS.

In this paper, we address this question by using the categorical approach and the more general language of finite tensor categories. This seems an appropriate approach, since the quasi-Frobenius property of an algebra is a Morita invariant property, and the Frobenius property is easily understood as well in terms of dimensions inside the category. This also allows us to include all the above-mentioned finite dimensional structures (Hopf algebras, quasi-Hopf algebras) and address the more general weak quasi-Hopf algebras. We show that a (finite dimensional) weak (or weak quasi) Hopf algebra $H$ is Frobenius if the dimensions of the matrix blocks of its base algebra $A$ are all equal. In particular, this approach recovers the well known results for Hopf and quasi-Hopf algebras. We also show that this is the best possible result, because if $A$ is a separable algebra over an algebraically closed field and the dimensions of the matrix blocks of $A$ are not all equal, then there is a weak Hopf algebra with base algebra $A$ which is not Frobenius. This is shown by constructing a tensor category $\mathcal{C}$ together with a tensor functor $F: \mathcal{C} \rightarrow \operatorname{Bimod}(A)$ into the category of $A$-bimodules (where $A$ is semisimple); then, applying general Tannakian reconstruction theory, we find the existence of a corresponding weak Hopf algebra which is not Frobenius.

\section{Preliminaries}

Throughout this paper, $K$ will be an algebraically closed field of characteristic 0 . We recall the following definition, for example, from $[\mathrm{BoSz}$,

Definition 1.1. A weak Hopf algebra is a $K$-vector space $H$ which is both an associative algebra $(H, m, 1)$ and a coassociative coalgebra $(H, \Delta, \varepsilon)$ together with an antipode $S: H \rightarrow H$ such that $\Delta: H \rightarrow H \otimes_{K} H$ is a morphism of algebras and

(i) $\varepsilon\left(a b_{(1)}\right) \varepsilon\left(b_{(2)} c\right)=\varepsilon(a b c)=\varepsilon\left(a b_{(2)}\right) \varepsilon\left(b_{(1)} c\right)$;

(ii) $1_{(1)} \otimes_{K} 1_{(2)} 1^{\prime}{ }_{(1)} \otimes 1^{\prime}{ }_{(2)}=1_{(1)} \otimes 1_{(2)} \otimes 1_{(3)}=1_{(1)} \otimes 1^{\prime}{ }_{(1)} 1_{(2)} \otimes 1^{\prime}(2)$, where $1^{\prime}=1$ is a "copy" of 1 ;

(iii) $a_{(1)} S\left(a_{(2)}\right)=\varepsilon\left(1_{(1)} a\right) 1_{(2)}$;

(iv) $S\left(a_{(1)}\right) a_{(2)}=1_{(1)} \varepsilon\left(a 1_{(2)}\right)$;

(v) $S\left(a_{(1)}\right) a_{(2)} S\left(a_{(3)}\right)=S(a)$.

If the counit is an algebra homomorphism, it is in fact just a Hopf algebra.

Also recall the definition of quasi-Hopf algebra [D], examples of which come from bialgebras with their comultiplication conjugated by a gauge element [D].

Definition 1.2. A quasi-bialgebra $H$ is an algebra with additional structure $(H, \Delta, \varepsilon, \Phi)$ where $\Delta: H \rightarrow H \otimes H$ is an algebra homomorphism and noncoassociative coproduct with counit augmentation $\varepsilon: H \rightarrow k$ satisfying the ordinary counit laws, $(\varepsilon \otimes \mathrm{Id}) \Delta=\mathrm{Id}=(\mathrm{Id} \otimes \varepsilon) \Delta . \Phi$ is an invertible element in $H \otimes H \otimes H$ denoted by $\Phi=X^{1} \otimes X^{2} \otimes X^{3}$ with inverse denoted by $\Phi^{-1}=x^{1} \otimes x^{2} \otimes x^{3}$ which controls the noncoassociativity of the coproduct on $H$ as follows:

$$
(\operatorname{Id} \otimes \Delta)(\Delta(a))=\Phi(\Delta \otimes \operatorname{Id})(\Delta(a)) \Phi^{-1} .
$$

Moreover, $\Phi$ must satisfy normalized 3-cocycle equations given by:

$$
\begin{gathered}
(1 \otimes \Phi)(\operatorname{Id} \otimes \Delta \otimes \operatorname{Id})(\Phi)(\Phi \otimes 1)=(\operatorname{Id} \otimes \operatorname{Id} \otimes \Delta)(\Phi)(\Delta \otimes \operatorname{Id} \otimes \operatorname{Id})(\Phi), \\
(\operatorname{Id} \otimes \varepsilon \otimes \operatorname{Id})(\Phi)=1 \otimes 1 .
\end{gathered}
$$


A quasi-bialgebra $H$ is a quasi-Hopf algebra if there is an anti-automorphism $S$ : $H \rightarrow H$, called an antipode, with elements $\alpha, \beta \in H$ such that $S\left(a_{(1)}\right) \alpha a_{(2)}=$ $\varepsilon(a) \alpha, a_{(1)} \beta S\left(a_{(2)}\right)=\varepsilon(a) \beta$ for all $a \in A$; moreover, $X^{1} \beta S\left(X^{2}\right) \alpha X^{3}=1$, and $S\left(x^{1}\right) \alpha x^{2} \beta S\left(x^{3}\right)=1$.

It was noted in $\mathrm{BoSz}$ that the category of finite dimensional representations of a weak Hopf algebra $H$ is a monoidal category, and moreover, it has a rigid structure; that is, it has left and right duals of every object, satisfying appropriate axioms. This has motivated the introduction of finite tensor categories. We recall the following definitions; we refer the reader to $\mathrm{BK}]$ or $\mathrm{CE}$ for technical details.

Definition 1.3. (i) An abelian category $\mathcal{C}$ is called finite if there are only finitely many isomorphism types of simples, each of which has a projective cover and objects have finite length and the Hom-spaces are finite dimensional. (Note: This is known to be equivalent to $\mathcal{C} \simeq \operatorname{Rep} A$ for a finite dimensional algebra $A$ ).

(ii) A category $\mathcal{C}$ which is $K$-linear abelian monoidal, rigid, all objects have finite length and the Hom spaces are finite dimensional vector spaces over $K$ is called a finite multitensor category. If $\mathbf{1}$ is simple, then this is called a finite tensor category.

(iii) A functor $F: \mathcal{C} \rightarrow \mathcal{D}$ between two monoidal categories is called quasi-tensor if there exist a natural isomorphism $\theta: F(X \otimes Y) \cong F(X) \otimes F(Y)$ for all $X, Y \in \mathcal{C}$, and $F\left(1_{\mathcal{C}}\right) \cong 1_{\mathcal{D}}$. This is called a tensor functor if the isomorphism $\theta$ agrees with the associativity and unit isomorphisms of $\mathcal{C}$ and $\mathcal{D}$.

It has been proven in $[\mathrm{EO}$ (see also $[\mathrm{HO}$ ) that any finite tensor category is equivalent to the representation theory of a weak quasi-Hopf algebra: we refer to MS for the technically precise definition of this generalization of weak Hopf algebra and quasi-Hopf algebra not needed in this paper (although the counit is not required to be an algebra homomorphism). This is done as follows: first one constructs a quasi-tensor functor $F: \mathcal{C} \rightarrow \operatorname{Bimod}(A)$ to the category of finite dimensional $A$ bimodules of a semisimple algebra $A$. Then, it is shown that $\mathcal{C}$ is equivalent to the representation category of a certain weak quasi-Hopf algebra structure on $\operatorname{End}_{K}(F)$ (which is a weak Hopf algebra if $F$ is a tensor functor).

Conversely, if $\mathcal{C}=\operatorname{Rep} H$, the finite dimensional representations (left modules) of $H$, for a weak quasi-Hopf algebra $H$, one defines $A=A_{L}=\left\{\varepsilon\left(1_{(1)} a\right) 1_{(2)}, a \in A\right\}$ and $A_{R}=\left\{1_{(1)} \varepsilon\left(a 1_{(2)}\right), a \in A\right\}$. It turns out that $A_{R} \simeq\left(A_{L}\right)^{o p}$ via the antipode and $A_{L}$ and $A_{R}$ are semisimple and commute with each other; the algebra $A$ is called the base algebra of $H$. (The unit object $A$ in $H$-Mod, with action via projection formula implicit above, is simple, or irreducible, in many cases; e.g., for $H$ a connected groupoid algebra.) There exists a functor $F: \mathcal{C} \rightarrow A \otimes A^{o p}-\mathrm{Mod}=$ $\operatorname{Bimod}(A)$, which is a quasi-tensor functor. Moreover, if the algebra $H$ is a weak Hopf algebra, then $F$ is a tensor functor.

In order to distinguish the categorical duals coming from the rigidity axioms of a tensor category, we will use $V^{\vee}$ to denote the $K$-dual of the $K$-vector space $V$.

Definition 1.4. A finite dimensional algebra is called quasi-Frobenius if every (finitely generated) projective left (or, equivalently, right) $A$-module is injective and, equivalently, $A$ is an injective left (or right) $A$-module. A $K$-algebra is called Frobenius if and only if $A \simeq A^{\vee}$ are left (or, equivalently, as right) $A$-modules.

It has been shown in [V] Corollary 3.3] that a weak Hopf algebra is necessarily quasi-Frobenius; this result is obtained as a consequence of the integral theory and a 
Hopf module isomorphism for weak Hopf modules. This is also an easy consequence of [EO, Proposition 2.3], which provides a very short proof of this fact. Moreover, this proposition also implies the result for weak quasi-Hopf algebras. We use the tensor category philosophy to obtain results about the Frobenius property of weak Hopf algebras and weak quasi-Hopf algebras. We note that since $K$ is algebraically closed, a finite dimensional quasi-Frobenius algebra $A$ is Frobenius if and only if the socle and co-socle of any projective indecomposable $A$-module $P$ have the same dimension [L, 16.7-16.33]. Indeed, the multiplicity of $P$ in ${ }_{A} A$ is the same as the multiplicity of $S$, the co-socle $P / J(A) P$ of $P$, in $A / J(A)$, the semisimple residual algebra of $A$. This is exactly $\operatorname{dim}_{K}(S)$ since $K$ is algebraically closed ( $S$ is simple). The multiplicity of $P$ in $A^{\vee}$ is the same as the multiplicity of $Q=P^{\vee}$ and as the right $A$-module in $\left(A^{\vee}\right)^{\vee}=A_{A}$. Since $P$ is also injective, $Q=P^{\vee}$ is projective as the right $A$-module, and its multiplicity in $A_{A}$ is the dimension of the co-socle $T=Q / Q J(A)$ of $Q$. But $T^{\vee}$ is then the socle of $P$ by duality, and we get that the multiplicity of $P$ in $A$ and $A^{\vee}$ are the same iff $\operatorname{dim}(T)=\operatorname{dim}(S)$.

\section{Weak Hopf algebras and Frobenius properties}

Let $H$ be a weak quasi-Hopf algebra, and let $F: \mathcal{C}=\operatorname{Rep}(H) \rightarrow \operatorname{Bimod}(A)$ be the associated forgetful functor. Let $\left(V_{i}\right)_{i \in I}$ be the simple objects in $\mathcal{C}$ and let $P_{i}$ be their respective covers. The vector space dimension of an $H$-module $M$ is $\operatorname{dim}_{K}(F(M)$ ) (this is different from the categorical or Frobenius-Perron dimension). Then $H=\oplus_{i \in I} P_{i}^{\operatorname{dim}\left(F\left(V_{i}\right)\right)}$. Also denote by $\left(S_{j}\right)_{j=1, \ldots, p}$ the simple right $A$-modules; then $S_{i j}=S_{i}^{\vee} \otimes_{K} S_{j}$ are the simple $A$-bimodules. Let $d_{i}=\operatorname{dim}_{K}\left(S_{i}\right)$. We recall that each object $X$ of $\mathcal{C}$ has an associated matrix $N_{X j}^{k}$ defined by multiplying the left-hand side by $X$, where $N_{X j}^{k}$ is the multiplicity of $V_{k}$ in the Jordan-Hölder series of $X \otimes L_{j}$ in $\mathcal{C}$. As in [EO, Section 2.8], for each projective $P_{i}, i \in I$, let $D(i) \in I$ be such that $P_{i}^{*} \simeq P_{D(i)}$ (here $(-)^{*}$ denotes the categorical right dual). Also, there is an invertible object $V_{\rho}$ of $\mathcal{C}$ such that $P_{D(i)}=P_{*}{ }_{i} \otimes V_{\rho}$ and $V_{D(i)}=V_{{ }_{*} i} \otimes V_{\rho}={ }^{*} V_{i} \otimes V_{\rho}$, where we convey $V_{{ }^{*} i}={ }^{*} V_{i}$.

Proposition 2.1. Denote by $\left[F(X): S_{i j}\right]$ the multiplicity of $S_{i j}$. Then

$$
\begin{aligned}
\operatorname{dim}_{K}\left(F\left(\operatorname{soc}\left(P_{k}\right)\right)\right) & =\sum_{i, j}\left[F\left(V_{D(k)}\right): S_{i j}\right] d_{i} d_{j} \\
\operatorname{dim}_{K}\left(F\left(\operatorname{cosoc}\left(P_{k}\right)\right)\right) & =\sum_{i, j}\left[F\left({ }^{*} V_{k}\right): S_{i j}\right] d_{i} d_{j} .
\end{aligned}
$$

Proof. We have $P_{k}^{*} \rightarrow \operatorname{cosoc}\left(P_{k}^{*}\right) \rightarrow 0$. Equivalently, by taking left duals, we get $0 \rightarrow{ }^{*} \operatorname{cosoc}\left(P_{k}^{*}\right) \rightarrow{ }^{*}\left(P_{k}^{*}\right)=P_{k}$, so $\operatorname{soc}\left(P_{k}\right)={ }^{*} \operatorname{cosoc}\left(P_{k}^{*}\right)$. Also, $\operatorname{dim}_{K}\left(F\left({ }^{*} X\right)\right)=$ $\operatorname{dim}_{K}\left(F\left(X^{*}\right)\right)=\operatorname{dim}_{K}(F(X))^{\vee}=\operatorname{dim}_{K}(F(X))$ (in $\operatorname{Bimod}(A)$, left and right duals are the same). Therefore

$$
\begin{aligned}
\operatorname{dim}_{K}\left(F\left(\operatorname{soc}\left(P_{k}\right)\right)\right) & =\operatorname{dim}_{K}\left(F\left({ }^{*} \operatorname{cosoc}\left(P_{k}^{*}\right)\right)\right) \quad(\text { by duality }) \\
& =\operatorname{dim}_{K}\left(F\left({ }^{*} \operatorname{cosoc}\left(P_{D(k)}\right)\right)\right) \quad\left(P_{D(k)} \simeq P_{k}^{*}\right) \\
& =\operatorname{dim}_{K}\left(F\left({ }^{*} V_{D(k)}\right)\right)=\operatorname{dim}_{K}\left(F\left(V_{D(k)}\right)\right) \\
& =\sum_{i, j}\left[F\left(V_{D(k)}\right): S_{i j}\right] d_{i} d_{j} .
\end{aligned}
$$

The second equality follows similarly. 
If $X, Y$ are objects of $\mathcal{C}$, then the matrix $M_{X}=\left[F(X): S_{i j}\right]_{i, j=1, \ldots, n}$ has integer coefficients, and moreover $M_{X \otimes Y}=M_{X} M_{Y}$. Indeed, if $F(X)=\bigoplus_{i, j} \alpha_{i j} S_{i j}$ and $F(Y)=\bigoplus_{i, j} \beta_{i j} S_{i j}$, since $S_{i j} \otimes_{A} S_{k l}=\delta_{j k} S_{i l}$, we get $F(X) \otimes_{A} F(Y)=$ $\bigoplus_{i, j, k, l} \delta_{j k} \alpha_{i j} \beta_{k l} S_{i j} S_{k l}=\bigoplus_{i, l}\left(\sum_{k=1}^{p} \alpha_{i k} \beta_{k l}\right) S_{i l}$. With this we have:

Theorem 2.2. Let $H$ be a finite dimensional weak quasi-Hopf algebra with the base algebra $A$. If the dimensions of the simple components of $A$ are all equal, then $H$ is a Frobenius algebra. In particular, this is true if the base algebra $A$ is commutative and when $H$ is a quasi-Hopf algebra.

Proof. Since $V_{D(k)}={ }^{*} V_{k} \otimes V_{\rho}, M_{V_{D(k)}}=M_{*} V_{k} \cdot M_{V_{\rho}}$. But since $V_{\rho}$ is invertible, $M_{V_{\rho}} \cdot M_{V_{\rho}^{-1}}=M_{V_{\rho}^{-1}} \cdot M_{V_{\rho}}=M_{V_{\rho} \otimes V_{\rho}^{-1}}=M_{1}=\mathrm{Id}$, so $M_{V_{\rho}}$ is a permutation matrix, since it has integer coefficients and its inverse $M_{V_{\rho}^{-1}}$ has integer coefficients also. So the columns of, and the elements of, $M_{V_{D(k)}}=\left[F\left(V_{D(k)}\right): S_{i j}\right]_{i, j=1, p}$ are a permutation of the columns of, and respectively the elements of, $M_{*} V_{k}=$ $\left[F\left({ }^{*} V_{k}\right): S_{i j}\right]$. Thus, if $d=d_{i}=d_{j}$ for all $i, j$ (for commutative $A, d=1$ ), using Proposition 2.1, one has

$$
\begin{aligned}
& \operatorname{dim}_{K}\left(F\left(\operatorname{soc}\left(P_{k}\right)\right)\right) \\
& \quad=d^{2} \sum_{i, j}\left[F\left(V_{D(k)}\right): S_{i j}\right]=d^{2} \sum_{i, j}\left[F\left({ }^{*} V_{k}\right): S_{i j}\right]=\operatorname{dim}_{K}\left(F\left(\operatorname{cosoc}\left(P_{k}\right)\right)\right)
\end{aligned}
$$

and so $H$ is Frobenius.

Remark 2.3. For the above proposition, we do not need that the characteristic of $K$ is 0 , since for any weak quasi-Hopf algebra, one can build a forgetful functor $F: \operatorname{Rep}(H) \rightarrow \operatorname{Bimod}(A)$.

Example 2.4. Let $B$ be Taft's Hopf algebra of dimension $p^{2}$, with generators $g, x$ with $g^{p}=1, x^{p}=0, x g=\lambda g x$ with $\lambda$ a primitive $p$ th root of unity, and comultiplication $\Delta(g)=g \otimes g, \Delta(x)=g \otimes x+x \otimes 1$, counit $\varepsilon(g)=1, \varepsilon(x)=0$, and antipode $S(g)=g^{-1}, S(x)=-g^{-1} x$. Let $x_{k}^{(s)}=\sum_{i=0}^{p-1} \lambda^{-i k} g^{i} x^{s}$, where we agree to write all indices modulo $p$. Note that

$$
\begin{aligned}
& g \cdot x_{k}^{(s)}=\lambda^{k} x_{k}^{(s)}, \\
& x \cdot x_{k}^{(s)}=x_{k-1}^{(s+1)} .
\end{aligned}
$$

Denote $V_{k}$ as the one dimensional $B$-module $K$ with structure $x \cdot \alpha=0$ and $g \cdot \alpha=$ $\lambda^{k} \alpha$. These form a set of representatives for simple left $B$-modules.

Let $I_{k}^{i}=B \cdot x_{k}^{i}={ }_{K}\left\langle x_{k+1}^{(p-1)}, x_{k+2}^{p-2}, \ldots, x_{k+i+1}^{(p-i)}\right\rangle(i=1, \ldots, p)$, the $K$-subspace spanned by these $i$ elements, which are linearly independent (since they contain different powers of $x$ ). There is a Jordan-Hölder series of $I_{k}^{k}, 0 \subseteq I_{k}^{1} \subseteq I_{k}^{2} \subseteq \cdots \subseteq$ $I_{k}^{k-1} \subseteq I_{k}^{k}$, and the terms of these series are $I_{k}^{i} / I_{k}^{i-1} \simeq V_{k+i}$ by (4). We have $J(B)=$ $B \cdot x$, the Jacobson radical of $B$. Then $J(B) \cdot I_{k}^{k}=I_{k}^{k-1}$ is the Jacobson radical of $I_{k}^{k}$, and so we have a superfluous morphism $I_{k}^{k} \rightarrow V_{k} \rightarrow 0$ (i.e. an epimorphism whose kernel is small). If $P_{k}$ is the projective cover of $V_{k}$, it follows that $P_{k}$ projects onto $I_{k}^{k}$, so it has dimension at least $p$. Since each projective indecomposable $P_{k}$ occurs in a decomposition of ${ }_{B} B$, we have that $p^{2}=\operatorname{dim}_{K}(B) \geq \sum_{i=0}^{p-1} \operatorname{dim}_{K}\left(P_{i}\right) \geq p \cdot p$, and therefore $\operatorname{dim}\left(P_{k}\right)=p$ for all $k$. Thus $P_{k}=I_{k}^{k}$. In fact, since $I_{k}^{i-1}=J(B) \cdot I_{k}^{i}$, we can see that each $P_{k}$ is a chain module (the $I_{k}^{i}$ are the only submodules). However, 
$P_{k}$ is also injective and has simple socle, and $\operatorname{soc}\left(P_{k}\right)=I_{k}^{1} \simeq V_{k+1}$. We now build a tensor functor $F: \operatorname{Rep}(B) \rightarrow \operatorname{Bimod}(A)$ in several steps:

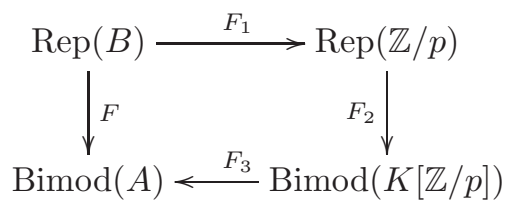

First, let $F_{1}: \operatorname{Rep}(B) \rightarrow \operatorname{Rep}(\mathbb{Z} / p)$ be the forgetful functor, given by the inclusion of Hopf algebras $\left\langle 1, g, \ldots, g^{p-1}\right\rangle \simeq K[\mathbb{Z} / p] \hookrightarrow B$. It is easily checked that this is a tensor functor. Let $F_{2}: K[\mathbb{Z} / p]-\bmod =\operatorname{Rep}(\mathbb{Z} / p) \rightarrow \operatorname{Bimod}(K[\mathbb{Z} / p]), F_{2}\left(V_{k}\right)=$ $\bigoplus_{i+j=k} V_{i}^{*} \otimes_{K} V_{j}=\bigoplus_{i} V_{-i} \otimes_{K}\left(V_{-i} \otimes V_{k}\right)$, where the second tensor represents the tensor product in $\operatorname{Rep}(\mathbb{Z} / p)$, and $(-)^{*}$ is the dual in $\operatorname{Rep}(\mathbb{Z} / p)$. On morphisms $f: X \rightarrow Y$, we have $F_{2}(f)=\bigoplus_{i=0}^{p-1} 1_{V_{i}^{*}} \otimes_{K}\left(1_{V_{-i}} \otimes f\right)$. It can be easily noted that $F_{2}$ is well defined (the action of $K[\mathbb{Z} / p] \otimes K[\mathbb{Z} / p]$ on $V_{i} \otimes_{K} V_{j}$ is on components) and that $F_{2}$ is a tensor functor: indeed, it is enough to check this on simple objects

$$
\begin{aligned}
F_{2}\left(V_{a}\right) \otimes_{K[\mathbb{Z} / p]} F_{2}\left(V_{b}\right) & =\left(\bigoplus_{i+j=a} V_{-i} \otimes V_{j}\right) \otimes_{K[\mathbb{Z} / p]}\left(\bigoplus_{k+l=b} V_{-k} \otimes V_{l}\right) \\
& =\bigoplus_{i, j, k, l ; i+j=a, k+l=b} \delta_{j,-k} V_{-i} \otimes V_{l} \\
& =\bigoplus_{i+l=a+b} V_{-i} \otimes V_{l}=F_{2}\left(V_{a} \otimes V_{b}\right) .
\end{aligned}
$$

Note that this functor can also be seen as the left adjoint of the functor

$$
G: \operatorname{Bimod}(\mathbb{Z} / p)=\operatorname{Rep}(\mathbb{Z} / p \times \mathbb{Z} / p) \longrightarrow K[\mathbb{Z} / p]-\bmod =\operatorname{Rep}(\mathbb{Z} / p),
$$

which is induced by the diagonal map $K[Z / p] \rightarrow K[Z / p] \otimes K[Z / p]$ coming from the group morphism $\mathbb{Z} / p \ni i \mapsto(-i, i) \in \mathbb{Z} / p \times \mathbb{Z} / p$. Indeed, we can easily see that $G\left(V_{a}^{*} \otimes_{K} V_{b}\right)=V_{a+b}$, so

$$
\begin{gathered}
\operatorname{Hom}_{\operatorname{Bimod}(\mathbb{Z} / p)}\left(F_{2}\left(V_{k}\right), V_{a}^{*} \otimes_{K} V_{b}\right) \\
=\operatorname{Hom}_{\operatorname{Bimod}(\mathbb{Z} / p)}\left(\bigoplus_{i+j=k} V_{i}^{*} \otimes_{K} V_{j}, V_{a}^{*} \otimes_{K} V_{b}\right)=\bigoplus_{i+j=k} \delta_{i, a} \delta_{j, b} K \\
=\delta_{a+b, k} K=\operatorname{Hom}_{K[\mathbb{Z} / p]-\bmod }\left(V_{k}, V_{a+b}\right)=\operatorname{Hom}_{K[\mathbb{Z} / p]-\bmod }\left(V_{k}, G_{V_{a}^{*} \otimes_{K} V_{b}}\right) .
\end{gathered}
$$

Finally, let $d_{1}, \ldots, d_{p}$ be positive integers, $A=\bigoplus_{i=1}^{p} M_{d_{i}}(K), F_{3}: \operatorname{Bimod}(\mathbb{Z} / p) \rightarrow$ $\operatorname{Bimod}(A), F_{3}\left(V_{i}^{*} \otimes V_{j}\right)=S_{i}^{\vee} \otimes S_{j}=S_{i j}$ (with $S_{i}$ 's as before). This is actually an equivalence of tensor categories. Let $F=F_{3} \circ F_{2} \circ F_{1}: \operatorname{Rep}(B) \rightarrow \operatorname{Bimod}(A)$, which is a tensor functor. By the above, using Tannakian reconstruction, this corresponds to a weak Hopf algebra $H=\operatorname{Taft}\left(d_{1}, \ldots, d_{n}\right)$ with base $A$ and $\operatorname{Rep}(H) \simeq \operatorname{Rep}(B)$, and "forgetful" functor the $F$ above. This holds in characteristic different from 0 , whenever none of the $d_{i}$ are divisible by the characteristic of $K$.

Proposition 2.5. With the notation above, the weak Hopf algebra $H$ is a Frobenius algebra if and only if $d_{1}, \ldots, d_{p}$ are all equal. Also, the algebra $H$ has dimension $\left(\sum_{i} d_{i}\right)^{4}$. Thus, if the $d_{i}$ 's are not all equal, $H$ is a weak Hopf algebra which is not a Frobenius algebra. 
Proof. By the considerations above we have

$$
\operatorname{dim}_{K}\left(F\left(\operatorname{soc}\left(P_{k}\right)\right)\right)=\operatorname{dim}_{K}\left(F\left(V_{k+1}\right)\right)=\operatorname{dim}_{K}\left(\bigoplus_{i+j=k+1} S_{i}^{\vee} \otimes S_{j}\right)=\sum_{i+j=k+1} d_{i} d_{j}
$$

and

$$
\operatorname{dim}_{K}\left(F\left(\operatorname{cosoc}\left(P_{k}\right)\right)\right)=\operatorname{dim}_{K}\left(F\left(V_{k}\right)\right)=\sum_{i+j=k} d_{i} d_{j} .
$$

$H$ is Frobenius if and only if these two numbers are equal for all $k$. Let $\omega$ be a root of order $p$ of unity different from 1 , and let $t(x)=\sum_{k=0}^{p-1} d_{k} x^{k}$. Then

$$
t(\omega)^{2}=\sum_{i, j} d_{i} d_{j} \omega^{i+j}=\sum_{k=0}^{p-1} \sum_{i+j=k} d_{i} d_{j} \omega^{k}=\left(\sum_{i} d_{i} d_{-i}\right) \cdot\left(\sum_{k} \omega^{k}\right)=0
$$

(the indices are always considered $\bmod p$ ). Therefore, $t$ is divisible by the polynomial $\sum_{k=0}^{p-1} x^{p}$, and so they are a multiple of each other. This implies that all $d_{i}$ are equal.

Since every projective indecomposable $P_{k}$ has each simple object occurring exactly once in any of its Jordan-Hölder series,

$$
\begin{aligned}
\operatorname{dim}_{K}(H) & =\sum_{k} \operatorname{dim}_{K}\left(F\left(P_{k}\right)\right) \cdot \operatorname{dim}_{K}\left(F\left(\operatorname{soc}\left(P_{k}\right)\right)\right) \\
& =\sum_{k}\left(\sum_{i} \operatorname{dim}_{K}\left(F\left(V_{i}\right)\right)\right) \cdot \operatorname{dim}_{K}\left(F\left(V_{k}\right)\right) \\
& =\left(\sum_{k} \operatorname{dim}_{k}\left(F\left(V_{k}\right)\right)\right)^{2}=\left(\sum_{k} \sum_{i+j=k} d_{i} d_{j}\right)^{2} \\
& =\left(\sum_{k} d_{k}\right)^{4} .
\end{aligned}
$$

However, with the observation above on the characterization of Frobenius algebras, there is a certain categorical statement which could be interpreted as the analogue of the property of (quasi) Hopf algebras is Frobenius:

Proposition 2.6. If $\mathcal{C}$ is a finite tensor category, then $d_{+}\left(\operatorname{soc}\left(P_{k}\right)\right)=d_{+}\left(\operatorname{cosoc}\left(P_{k}\right)\right)$, where $d_{+}$represents the Frobenius-Perron dimension in $\mathcal{C}$.

Proof. As in Proposition 2.1, $\operatorname{soc}\left(P_{k}\right)={ }^{*} L_{D(k)}$. We compute

$$
\begin{aligned}
d_{+}\left(\operatorname{soc}\left(P_{k}\right)\right) & =d_{+}\left({ }^{*} L_{D(k)}\right)=d_{+}\left(L_{D(k)}\right)=d_{+}\left({ }^{*} L_{k} \otimes L_{\rho}\right) \\
& =d_{+}\left({ }^{*} L_{k}\right) d_{+}\left(L_{\rho}\right)=d_{+}\left(L_{k}\right)=d_{+}\left(\operatorname{cosoc}\left(P_{k}\right)\right) .
\end{aligned}
$$

Note that Proposition 2.6 implies the Hausser-Nill result that quasi-Hopf algebras are Frobenius algebras.

One can ask whether a weak (quasi-) Hopf algebra $H$ is perhaps Frobenius "over its base algebra" $A$; this should naturally be formulated in the terminology of Frobenius extensions. If $\varphi: A \rightarrow B$ is a morphism (extension) of rings, it is called a Frobenius extension if the forgetful (restriction of scalars) functor ${ }_{B} \mathcal{M} \rightarrow{ }_{A} \mathcal{M}$ has isomorphic left and right adjoints (see $[\mathbf{K}]$ for details). We have the extension 
of algebras $A \hookrightarrow H$, and one can ask the question whether this is Frobenius. Since $A$ is semisimple, the unit $k \rightarrow A$ is a Frobenius extension, and then if $A \hookrightarrow H$ is Frobenius, $k \hookrightarrow H$ would be Frobenius (by transitivity of Frobenius extensions). But, as seen above, this is not always the case. This provides an example of a (finite projective) weak Hopf-Galois extension which is not a Frobenius extension, since $H$ is such a Galois extension of $A$ CDG, 2.7]. (Similarly, the extensions $A \hookrightarrow H$ and $A \otimes A^{o p}=A_{L} \otimes A_{R} \hookrightarrow H$ are not twisted or $\beta$-Frobenius extensions, although they are QF extensions since the base algebras are separable.)

\section{Acknowledgments}

The first author wishes to thank Pavel Etingof from MIT for his great support and the excellent time spent at MIT during the fall semester of 2008. This paper would not have been possible without his contribution, as an important part of the ideas contained here are actually to his credit. The authors also thank Kornel Szlachányi and colleagues in Budapest for comments about this paper.

\section{REFERENCES}

[BC] D. Bulacu, S. Caenepeel, Integrals for (dual) quasi-Hopf algebras. Applications, J. Algebra 266 (2003), no. 2, 552-583. MR1995128 (2004k:16095)

[BNS] G. Böhm, F. Nill, K. Szlachányi, Weak Hopf algebras. I. Integral theory and $C^{*}$-structures, J. Algebra 221 (1999), 385-438. MR1726707 (2001a:16059)

[BoSz] G. Böhm, K. Szlachányi, A coassociative $C^{*}$-quantum group with nonintegral dimensions, Lett. Math. Phys. 38 (1996), no. 4, 437-456. MR1421688 (97k:46080)

[BK] B. Bakalov, A. Kirillov, Jr., Lectures on tensor categories and modular functors, Univ. Lect. Ser. 21, Amer. Math. Soc., Providence, RI, 2001. MR1797619 (2002d:18003)

[CE] D. Calaque, P. Etingof, Lectures on tensor categories, in Quantum Groups (B. Enriquez, V. Turaev, eds.), IRMA Lectures in Mathematics and Theoretical Physics, 12, EMS, 2008. MR2432988 (2009g:18010)

[CDG] S. Caenepeel, E. De Groot, Galois theory for weak Hopf algebras, Rev. Roumaine Math. Pures Appl. 52 (2007), 151-176. MR2337749(2008g:16061)

[D] V.G. Drinfel'd, Quasi-Hopf algebras, Leningrad Math. J. 1 (1990), 1419-1457. MR.1047964 (91b:17016)

[EO] P. Etingof, V. Ostrik, Finite tensor categories, Mosc. Math. J. 4, No. 3 (July-Sept. 2004), 627-654. MR 2119143 (2005j:18006)

[ENO] P. Etingof, D. Nikshych, V. Ostrik, On fusion categories, Ann. of Math. (2) 162 (2005), no. 2, 581-642. MR2183279 (2006m:16051)

[FFRS] J. Frölich, J. Fuchs, I. Runkel, C. Schweigert, Picard groups in rational conformal field theory, Contemp. Math., 391, Amer. Math. Soc., Providence, RI, 2005, 85-100. MR2184014 (2007a:81131)

[HO] R. Haring-Oldenburg, Reconstruction of weak quasi-Hopf algebras, J. Algebra 194 (1997), no. 1, 14-35. MR:1461480 (98h:16062)

[K] L. Kadison, New Examples of Frobenius Extensions, Univ. Lect. Ser., 14, Amer. Math. Soc., Providence, RI, 1999. MR1690111 (2001j:16024)

[L] T.-Y. Lam, Lectures on Modules and Rings, Grad. Texts in Math., 189, Springer, 1999. MR:1653294 (99i:16001)

[MS] G. Mack, V. Schomerus, Quasi-Hopf quantum symmetry in quantum theory, Nuclear Phys. B 370 (1992), no. 1, 185-230. MR 1148544 (93c:81081)

[N] D. Nikshych, On the structure of weak Hopf algebras, Adv. Math. 170 (2002), 257-286. MR $1932332(2003 f: 16063)$ 
[S] K. Szlachányi, Weak Hopf algebras, Operator algebras and quantum field theory (Rome, 1996), 621-632, Int. Press, Cambridge, MA, 1997. MR1491146 (99a:46105)

[V] P. Vecsernyés, Larson-Sweedler theorem and the role of grouplike elements in weak Hopf algebras, J. Algebra 270 (2003), 471-520. MR2019628 (2005a:16058)

Faculty of Mathematics, University of Bucharest, Str. Academiei 14, RO-70109, Bucharest, Romania - And - State University of New York, Buffalo, 244 Mathematics Building, Buffalo, New York 14260-2900

E-mail address: yovanov@gmail.com, e-mail@yovanov.net

Department of Mathematics, University of Pennsylvania, David Rittenhouse Laboratory, 209 S. 33rd Street, Philadelphia, Pennsylvania 19104

E-mail address: lkadison@math.upenn.edu

Current address: Department of Mathematics, University of California at San Diego, 9500 Gilman Drive, \#0112, La Jolla, California 92093 